\title{
The Effect of Habitat Fragmentation on Cyclic Populations with Edge Behaviour
}

\author{
T. Gauduchon ${ }^{1}$, S. Strohm², R.C. Tyson ${ }^{3} *$ \\ ${ }^{1}$ Agrocampus Ouest, 65 rue de Saint Brieuc, CS84215 - 35042 Rennes Cedex, France \\ ${ }^{2}$ Mathematics and Statistics, Okanagan College, 583 Duncan Ave W, Penticton, BC, V2A 8E1 Canada \\ ${ }^{3}$ Mathematics and Statistics, IKBSAS 5 SCI, University of British Columbia Okanagan, 3333 University \\ Way, Kelowna, BC, V1V 1V7, Canada
}

\begin{abstract}
Habitat fragmentation is an important area of concern in species conservation. Habitat fragmentation can affect population distributions through reductions in suitable habitat, and through organism responses to different habitat types and the transitions between them. In earlier work, the effect of habitat fragmentation on cyclic populations was investigated in the context of populations that show no behavioural response to the interface between habitat types. In this paper, we extend the earlier work by adding edge-mediated behaviour to the models. That is, we investigate the dynamics that result when oscillatory predator and prey species also exhibit behavioural responses to habitat interfaces. Our results show generally that habitat loss decreases the amplitude and the average density of the prey and predator populations, but that most of the reponses observed in the two models exhibit marked differences. This work highlights the complexity of the interplay between population cycles, habitat fragmentation, and edge-mediated behaviour, and the need to study such systems in greater detail.
\end{abstract}

Keywords and phrases: Rosenzweig-MacArthur model, May model, habitat fragmentation, mathematical model, predator-prey, cyclic populations

Mathematics Subject Classification: 92B99, 37N25, 65Mxx

\section{Introduction}

Habitat fragmentation is an important area of concern in species conservation [27]. Many empirical and mathematical studies have examined the effect of habitat fragmentation for a range of species and behaviours $[2,25,26,28,29,33]$. Habitat fragmentation can affect species in a number of different ways. Here, we are particularly interested in the effects of fragmentation on organism movement. The idea that characteristics of individual organism movement can be habitat-dependent is intuitive, and has been observed in field studies (see [17] for a discussion). The consequences of making movement parameters habitat-dependent have been explored in a number of mathematical studies [19,32].

In addition to movement patterns specific to different habitats, ecologists have also noticed that edges between habitats generate another range of edge-specific behaviours. Haughland and Larsen [10] found that dispersing juvenile red squirrels born in the edge region between mature and thinned forest, settled

${ }^{*}$ Corresponding author. E-mail: rebecca.tyson@ubc.ca

(C) EDP Sciences, 2013 
in edge regions even though they recognized the higher quality of mature forest and biased their searches for vacant territories in that direction. Villis [33] looked at the effect of edge shape on edge permeability for meadow voles and studied movements of butterflies at the edge of a fragmented landscape. This study was able to explain butterfly distributions with the behavior-at-boundary hypothesis, which assumes a patch preference for suitable habitat patches. In recent studies, patch preferences have been shown for several mammals of the boreal forests: the wolf [9], the wolverine [11] and the lynx [14]. Global Positioning System (GPS) data-gathering from radio-monitored animals has clearly shown the habitat selection made by these species.

Since these organisms show preference for particular habitats, they must respond to habitat edges. Upon reaching a habitat boundary, possible responses include quickly crossing the matrix to reach the next preferred patch, moving away from the boundary in the direction of the preferred habitat, or ignoring the boundary altogether. In each case, the organism can demonstrate differential movement rates in each patch, and/or different levels of preference for the patches on either side of the interface. Each pattern of individual responses to habitat edges dictates a particular mathematical boundary condition [17,23], and this boundary condition in turn affects the population distribution within and across patches as well as important quantities such as critical patch size [17].

A logical extension of previous work $[17,23]$ is to ask how edge behaviour affects the dynamics of cyclic populations. Multi-annual cyclic population dynamics are widespread in nature [1,20]. Famous examples include the snowshoe hare (Lepus americanus) with its specialist predator the Canada lynx (Lynx canadensis) [16], and the meadow vole (Microtus agretis) with its specialist predator the least weasel (Mustela nivalis) [34]. Cyclic species, like non-cyclic ones, also exhibit preference for certain habitats. In Washington State, for example, lynx prefer Engelmann spruce (Picea engelmannii) and sub-alpine fir (Abies lasiocarpa) forests with moderate canopy cover [14].

The effects of habitat fragmentation on cyclic population dynamics were recently studied by Strohm and Tyson [29]. In their work, a theoretical examination of habitat loss, habitat separation, and separation and loss combined, showed that habitat fragmentation affects the amplitude and average density of cyclic predator-prey populations. The movement rates in this study were habitat-dependent, with higher diffusion in matrix habitat. The boundary conditions however, were the continuous population density and flux conditions classically used in the dispersal literature. These are precisely the boundary conditions that assume no response by the organism to habitat edges. In this study, we extend our previous work by examining the effect of organism response to habitat edges. We find that the response of a cyclic system to decreasing good patch size varies with edge behaviour and the level of habitat-dependence in movement rates. In particular, we find that the response of populations in the middle and at the edge of the good patch can have opposite responses, with one population decreasing and the other increasing as fragmentation increases. For cyclic populations then, the effect of habitat fragmentation measured in one location can be misleading, and care must be taken in interpreting observed changes in population dynamics.

\section{The model}

There is a long mathematical modelling tradition in predator-prey models, and the model used by Strohm \& Tyson [29] follows this tradition. We use the same model: Predator and prey movement is modeled as random Fickian diffusion, and the two species are also subject to reaction terms describing population growth, death and predation. Using $n$ to represent prey density and $p$ to represent predator density, the dimensionless equations are

$$
\begin{aligned}
& \frac{\partial n}{\partial t}=D_{n}(i) \frac{\partial^{2} n}{\partial x^{2}}+f(n, p, x), \\
& \frac{\partial p}{\partial t}=D_{p}(i) \frac{\partial^{2} p}{\partial x^{2}}+g(n, p),
\end{aligned}
$$


where $i$ is the patch number, $D_{n}$ and $D_{p}$ are the patch-dependent diffusivity coefficients for prey and predator, respectively, and $f(n, p, x)$ and $g(n, p)$ are the reaction terms for prey and predator, respectively. The $x$-dependence in the prey reaction terms arises through a spatially-dependent growth rate, see below. The diffusivity coefficients are patch-dependent, but constant within each patch.

For the reaction terms, Strohm \& Tyson used four different classical models, namely, the LotkaVolterra, Rosenzweig-MacArthur, May and Variable Territory models [29]. The Lotka-Volterra model is useful as a simple example of cyclic population dynamics, and was instrumental in demonstrating how the interactions between predator and prey can lead to multi-annual cycles $[6,15,22]$, but has structural properties that make it highly unrealistic as a model for real systems. We therefore do not consider this model in the present work. Also, since Strohm \& Tyson [29] found that the Variable Territory and Rosenzweig-MacArthur models generally exhibited the same response to habitat fragmentation, we only consider one of the two. In this paper, we thus consider the Rosenzweig-MacArthur and May models for the reaction terms $f(n, p, x)$ and $g(n, p, x)$.

Following Strohm \& Tyson [29], the May model is written

$$
\begin{aligned}
f(n, p, x) & =r(x) n\left(1-\frac{n}{k}\right)-\frac{c n p}{d+n}, \\
g(n, p) & =s p\left(1-\frac{q p}{n}\right)
\end{aligned}
$$

and the Rosenzweig-MacArthur model is written

$$
\begin{aligned}
f(n, p, x) & =r(x) n\left(1-\frac{n}{k}\right)-\frac{c n p}{d+n}, \\
g(n, p) & =\frac{\chi c n p}{d+n}-\delta p .
\end{aligned}
$$

Note that the prey equations are the same for the two models. The prey growth rate is logistic and limits prey densities to the carrying capacity of the region, and predation follows the classical specialist type II response. The two models differ in the predator equations. The Rosenzweig-MacArthur model encompasses growth through a biomass conversion of prey consumed (predation term). In contrast, the May model assumes that the growth of the predator is logistic with carrying capacity $n / q$ proportional to the prey density, $n$. This term in the model is based on the assumption that predator territory size increases with a decrease in the prey density. Unlike the Rosenzweig-MacArthur model, the May model does not incorporate direct biomass conversion from predator to prey.

We nondimensionalize the May model equations using

$$
\begin{aligned}
& n^{*}=\frac{n}{k}, \quad p^{*}=\frac{q p}{k}, \quad t^{*}=s t, \\
& r^{*}=\frac{r}{s}, \quad c^{*}=\frac{c}{q}, \quad d^{*}=\frac{d}{k}
\end{aligned}
$$

and the Rosenzweig-MacArthur model equations using

$$
\begin{aligned}
n^{*} & =\frac{n}{k}, & p^{*} & =\frac{p}{k \chi}, \\
r^{*} & =\frac{r}{\delta}, & t^{*} & =\delta t, \\
c^{*} & =\frac{\chi c}{\delta}, & d^{*} & =\frac{d}{k} .
\end{aligned}
$$

With these substitutions, and dropping asterisks for simplicity, the May reaction terms become

$$
\begin{aligned}
f(n, p, x) & =r(x) n(1-n)-\frac{c n p}{d+n}, \\
g(n, p) & =p\left(1-\frac{p}{n}\right)
\end{aligned}
$$


and the Rosenzweig-MacArthur reaction terms become

$$
\begin{aligned}
f(n, p, x) & =r(x) n(1-n)-\frac{c n p}{d+n}, \\
g(n, p) & =\frac{c n p}{d+n}-p .
\end{aligned}
$$

\subsection{A spatial model in a fragmented domain}

\subsubsection{The fragmented domain}

The spatial domain we consider in this paper is a 1-dimensional domain divided into three habitat patches where a good patch (preferred habitat) is placed between two bad habitat (matrix) patches. Numbering patches from left to right, patches 1 and 3 are therefore matrix, while patch 2 is preferred habitat. We maintain this numbering throughout the paper. As in [29], we assume that the birth rate $r(x)$ of the prey is zero in the bad patches and positive in the good patch. At the patch edges however, there is a narrow region where $r(x)$ increases rapidly and smoothly from 0 to the maximum value in the patch. This smoothing-out of $r(x)$ was necessary to prevent numerical difficulties, and was also used in [29]. Specifically, in good patches we used

$$
r(x)=r_{\max } \frac{\tan ^{-1}(l / 2-|x|)}{\tan ^{-1}(l / 2)} \quad \text { for }-l / 2 \leq x \leq l / 2
$$

where the good patch is centred at 0 and $l$ is the patch width. The boundaries of the domain are reflective (Neumann), that is, there is no movement outside the three-patch domain.

\subsubsection{The edge behavior}

At the interface between patches 1 and 2 (bad and good, respectively) we use the boundary conditions derived by Ovaskainen [23] and further explored by Maciel and Lutscher [17]. The first boundary condition is the usual flux conservation condition which ensures that the number of organisms crossing an interface at any moment is the same on both sides of the interface (that is, if 1 and 2 are neighboring patches, then the organisms leaving 1 must enter 2 and vice versa). We express this boundary condition mathematically as:

$$
D_{1} \frac{\partial u_{1}}{\partial x}=D_{2} \frac{\partial u_{2}}{\partial x}
$$

where,

$$
\begin{aligned}
D_{1}, D_{2} & =\text { the diffusivity coefficients in patches } 1 \text { and } 2 \\
u_{1}, u_{2} & =\text { the population densities at the edge of patches } 1 \text { and } 2 .
\end{aligned}
$$

Note that $u$ is a placeholder for the prey, $n$, or the predator, $p$. The formulation (2.11) allows for an organism's movement rate, $D$, to vary between patches. This is consistent with field studies showing that movement behaviours in matrix and habitat patches can differ $[3,4]$.

The second boundary condition arises from the pattern of individual responses to the boundary between the two patches, as well as the pattern of movement rates in patches with different resource levels. With respect to the latter aspect, we consider the case where each individual adjusts the probability of movement within a patch, in response to the resource density there, but keeps the stepsize constant. In other words, the wait time between each step is longer in resource-rich patches than in resource-poor ones, but the movement distance per step is the same in each patch. For details, please see [23]. While this is only an approximation to the possible interface response that a population of individuals may exhibit, it is nonetheless a plausible one $[5,24]$ and will serve our purpose of shedding some light on the effect of boundary behaviour on the dynamics of cyclic predator-prey populations. 
For the boundary response, we suppose that individuals can exhibit a preference for one patch over another, independent of individual movement rates in each patch. More specifically, at the patch 1-2 interface, we suppose that individuals at the interface move into patch 1 with probability $\alpha$ and patch 2 with probability $1-\alpha$. Using $u$ again as a placeholder for the prey or predator populations, the boundary condition is written

$$
u_{1}=\kappa u_{2}
$$

where

$$
\kappa=\frac{\alpha}{1-\alpha} \frac{D_{2}}{D_{1}} .
$$

For details, please see [23]. Following Maciel \& Lutscher [17], we refer to the parameter $\alpha$ as the preference term. A consequence of this boundary condition, is that the population density is not continuous across the boundary (i.e. $u_{1} \neq u_{2}$ if $\kappa \neq 1$. That is, if individuals exhibit a preference for one patch over another, there can be a discontinuous jump in the population density between patches 1 and 2. Edge behaviours consistent with a strong degree of preference for one patch over another have been observed in the field [3,10,12], as have strong differences in population densities on either side of an interface [4]. The expression (2.13) with (2.12) indicates that the discontinuity will increase in size as the organism's preference for one patch over the other increases (that is, $\alpha$ increases toward 1 or decreases toward zero). When $\alpha=1 / 2$, neither patch is preferred. If, in addition, $D_{1}=D_{2}$, the interface conditions are the same as in the previous work by Strohm et al [29].

Based on the ordering of good and bad patches in our simulations $(1=\operatorname{bad}, 2=$ good), when $\alpha=0$ all individuals choose to stay in the good patch (patch 2), and when $\alpha=1$ all individuals choose to stay in the bad patch (patch 1). We assume that predators and prey have a preference for the good patch (patch2) and so we can logically assume that

$$
u_{1}<u_{2} \quad \Leftrightarrow \quad 0<\kappa<1
$$

Combining (2.14) and (2.13), it therefore follows that

$$
0<\alpha<\frac{1}{\frac{D_{2}}{D_{1}}+1} .
$$

Parallel assumptions are true for the interface between patches 2 (good patch) and 3 (bad patch), so that the interface conditions are symmetric about the good patch.

\section{Simulation experiments}

\subsection{Habitat fragmentation and Movement Rates}

In the following, we present two broad classes of results. First, in Part I (Section 4) we examine the effect of decreasing good patch size on prey and predator average densities and cycle amplitudes. We observe how these vary when predator and prey have equal preference for the good patch, and when the movement rate of each species does not depend on the patch. In this section, population distributions across the interface result from the patch preferences or movement rates on either side, but not both. In Part II (Section 5), we hold good patch size constant and take a closer look at the effect of patch preference and habitat-dependent versus habitat-independent movement rates on population densities and amplitudes.

\subsection{Initial conditions and parameter values}

Prey and predator initial densities, $n_{0}$ and $p_{0}$, respectively, are constant over the domain at the beginning of the simulations. The parameter values used are taken from Strohm \& Tyson [29] and are shown in Table 1. Intuitively, one would expect that the parameter values would be the same between 


\begin{tabular}{|c|c|c|c|}
\hline Parameter & May & $\begin{array}{c}\text { Rosenzweig- } \\
\text { MacArthur }\end{array}$ & Description \\
\hline \hline$r$ & 1.75 & 2.1 & intrinsic rate of prey population increase \\
\hline$k$ & 8 & 11 & carrying capacity of prey \\
\hline$c$ & 505 & 800 & predator saturation kill rate \\
\hline$d$ & 0.3 & 1.5 & predator half-saturation constant \\
\hline$\chi$ & $\mathrm{n} / \mathrm{a}$ & 0.004 & prey-predator conversion rate \\
\hline$\delta$ & $\mathrm{n} / \mathrm{a}$ & 2.4 & predator death rate in the absence of prey \\
\hline$s$ & 0.85 & $\mathrm{n} / \mathrm{a}$ & intrinsic rate of predator population increase \\
\hline$q$ & 212 & $\mathrm{n} / \mathrm{a}$ & minimum prey biomass per predator \\
\hline$n 0$ & 275 & 5 & initial prey density \\
\hline$p 0$ & 5 & 0.015 & initial predator density \\
\hline
\end{tabular}

TABle 1. Parameter values for the May (2.2) and Rosenzweig-MacArthur (2.3) models.

the two models (for those parameters that the two models share), but that is not the case for our study here. This is because we are chiefly interested in understanding the change in characteristics of the cyclic dynamics as a result of fragmentation. Given a particular set of cycle probes such as amplitude, maximum, minimum and period, we find that in order to match these probes, it is necessary to use different parameters in the two models [29]. For our investigation then, we are using parameter values that give us approximately the same baseline dynamics in the absence of habitat fragmentation, as defined by the cycle probes.

\section{Results Part I: Habitat Loss}

For the results presented in this section, we investigate the effect of decreasing good patch size for different levels of prey and predator good patch preference, and different ratios of prey and predator diffusion rates. We assume however, that both prey and predator exhibit the same level of preference for the good patch $\left(\alpha_{\text {prey }}=\alpha_{\text {predator }}\right)$, and that each species has the same movement rate in the good and bad patches $\left(D_{n 1}=D_{n 2}\right.$ and $\left.D_{p 1}=D_{p 2}\right)$. We relax these assumptions in Section 5 .

A sample spatial profile of prey density is shown in Figure 1. In these plots, there is a strong preference for the good patch, and therefore there is a large discontinuity in population density at the interface between patches. At the patch sizes chosen, there are oscillations in all three patches, and at all locations within each patch. As a result of the temporal oscillations in the predator and prey densities throughout the simulation domain, the relative population densities in different locations vary with time. In the results below, we therefore present population densities in the middle and at the edge of the good patch averaged over at least one full period of the oscillation. Simulations were run until transients had disappeared, and then results obtained using the subsequent, steady-state oscillations.

\subsection{Prey only Results}

We first consider the effect of decreasing good patch size on the prey alone, that is, when predators are absent. This provides us with a baseline which we can use to interpret the coupled predator-prey results. The effects of habitat loss when the predator is absent are shown for three different values of $\alpha$ in Figure 2. Since the prey equation is the same for both models, the prey only results are also the same for both models. Consider first the effect of changes in $\alpha$. As $\alpha$ decreases, corresponding to stronger preference for the good habitat, the prey density in the bad patch decreases substantially (Figure 2, left panel). This is consistent with the notion that prey are increasingly likely (as $\alpha$ decreases) to choose the good patch when encountering the patch interface, thereby leading to a decrease in population density in the bad patch. At the same time, there is an increase in population density in the good patch, especially at the edge (Figure 2, middle panel). Finally, the changes in edge behaviour are much less apparent in the 

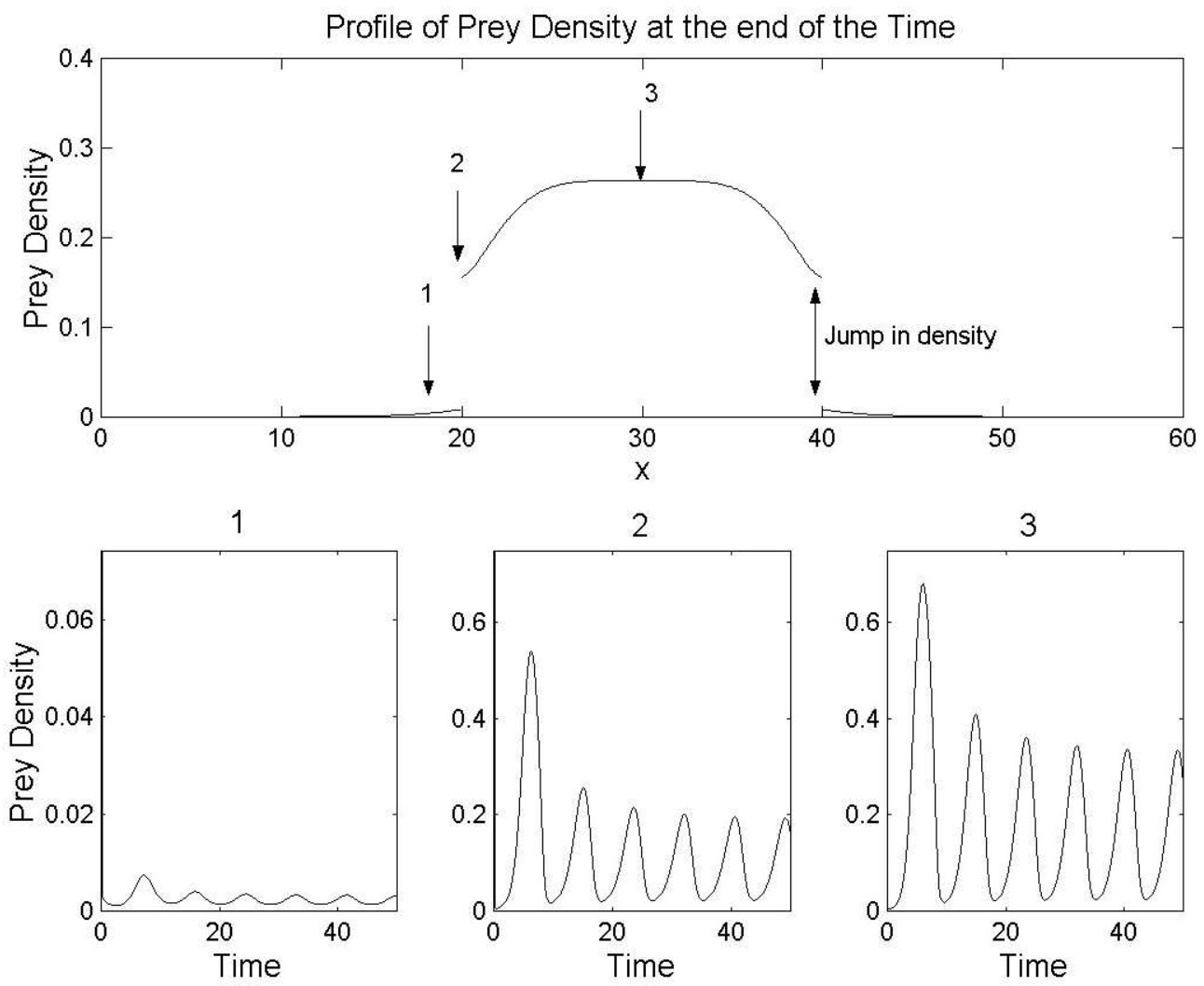

Figure 1. Spatial profile of the dimensionless prey density $(\alpha=0.05)$ (top) (predator density is not shown) after transients have disappeared. The three lower plots show the oscillations in density as a function of time at different locations in the spatial domain. These locations are indicated in the top figure. Here $l_{g}=l_{b}=20, D_{n 1}=D_{n 2}=1.5$, and $D_{p 1}=D_{p 2}=2.1$. The other parameter values are listed in Table 1 .

middle of the good patch where, for large patch sizes, the population density in the middle is essentially unaffected by patch preference (Figure 2, right panel).

Now consider the effect of habitat loss through decreasing good patch size, $l_{g}$. For a large range of $l_{g}$ values, there is no apparent effect of decreasing $l_{g}$. Eventually however, for $\alpha=0.5$ (no patch preference) and $\alpha=0.27$, the patch size decreases to the point where prey density drops off precipitously, and monotonically, even in the middle of the good patch. In fact, the effect of habitat loss is apparent in the middle of the good patch before it is observed at the edge. For $\alpha=0.05$ however, the prey has strong enough preference for the good patch that there is little loss to the matrix. Consequently, the loss of good habitat has very little effect on the population at the edge or in the middle of the good patch, even for very small values of $l_{g}$.

In the following sections, we consider the effect of habitat loss on the joint dynamics of predator and prey when both are present.

\subsection{Varying Patch Preference, $\alpha$}

A summary of the results for the May and Rosenzweig-MacArthur models is shown in Figure 3. For each case (middle or edge, may or Rosenzweig-MacArthur ) we show only the profile for prey average density. 

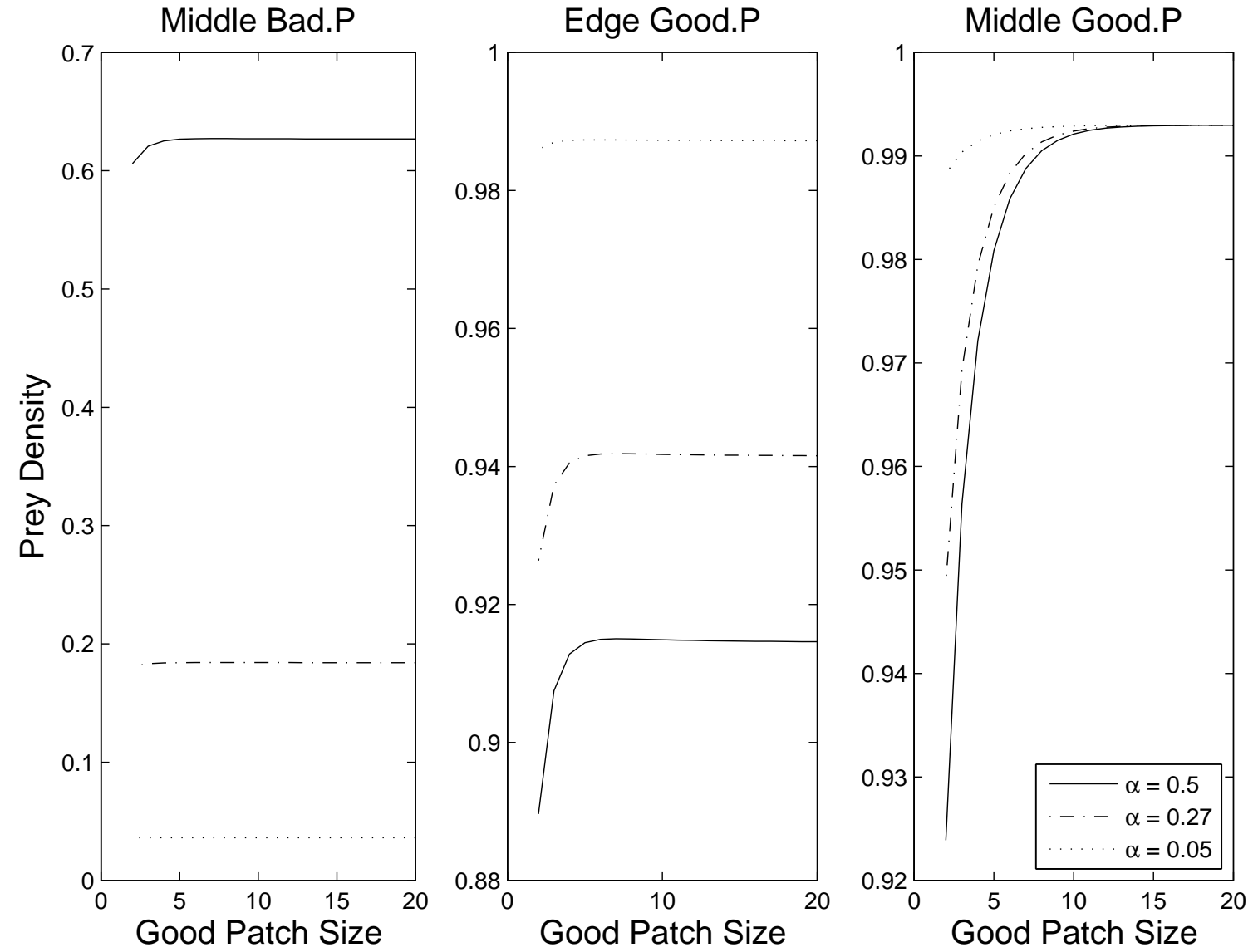

Figure 2. Plots of the dimensionless prey density against the good patch size after transients had disappeared. Density is plotted in the middle of the bad patch (Middle Bad P.), at the edge of the good patch (Edge Good P.), and in the middle of the good patch (Middle Good P.), for three different values of $\alpha$. To simplify the figure, the three plots share the same legend. Note that the $y$-axis labels are not the same. We take $D_{n 1}=D_{n 2}=1.5$ and $D_{p 1}=D_{p 2}=2.1$. Other parameters are listed in Table 1 .

The corresponding profiles for predator average density, prey amplitude and predator amplitude had the same shape. Prey average density therefore, is a proxy for predator average density, prey amplitude and predator amplitude as well.

We observe that in all four cases, the prey average density decreases with good patch size. For the May model, variations in patch preference, $\alpha$, have a stronger effect on the edge than in the middle of the good patch, while the effect is more comparable in both positions for the Rosenzweig-MacArthur model. When there is no patch preference, all but one population, the may model population at the edge of the good patch, initially show an increase in prey average density as good patch size decreases, before dropping precipitously once a minimum patch size is reached. The drop is more precipitous for the Rosenzweig-MacArthur model than for the May model.

The May population at the edge of the good patch is the only one for which prey density increases as patch preference increases, for all good patch sizes tested. The same behaviour is exhibited by the May population in the middle of the good patch when the good patch is very small $\left(l_{g}<4\right)$, and possibly also for the largest patch size $\left(l_{g}=20\right)$, though the difference is minimal. In general, increased patch 

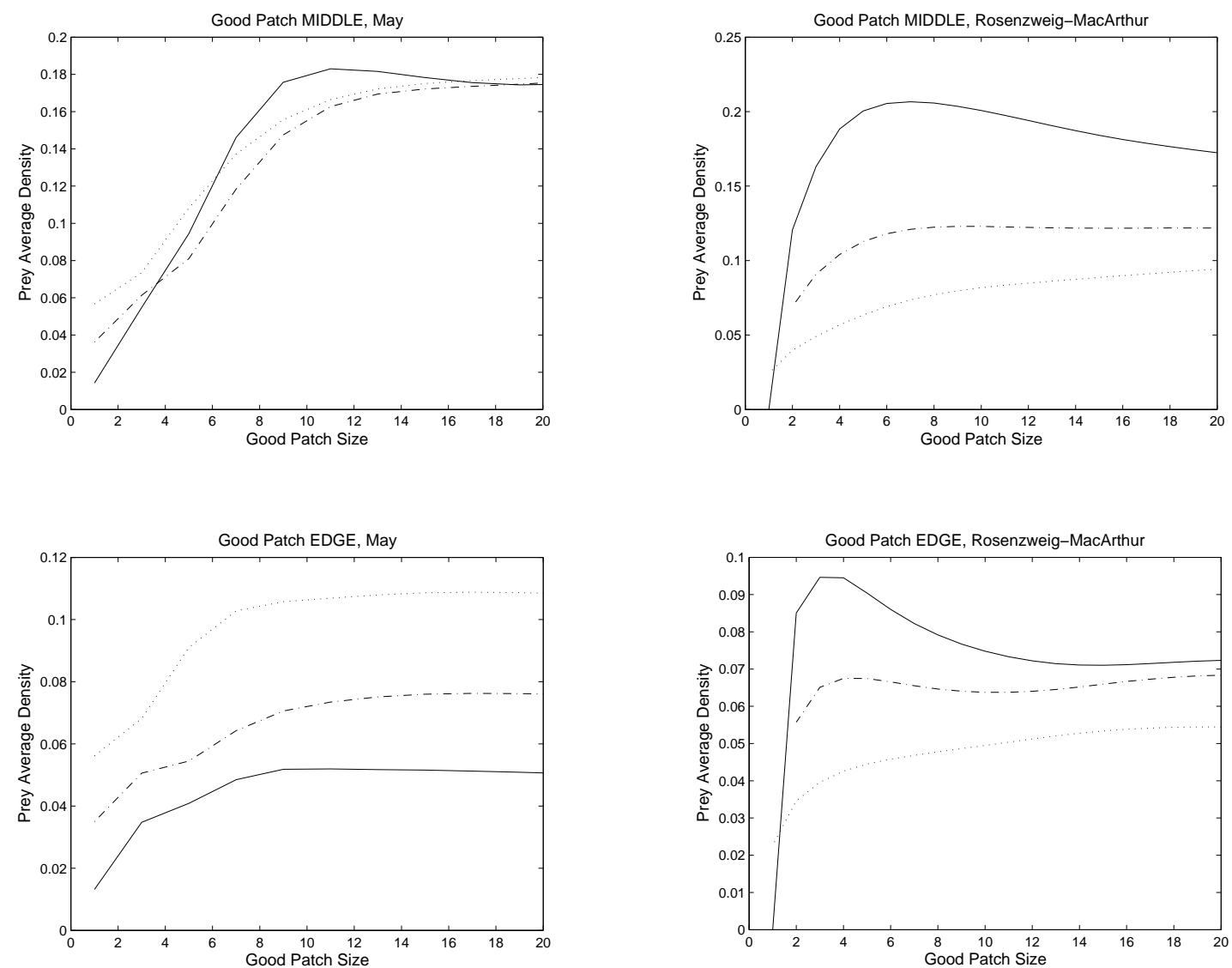

$$
\alpha=0.5
$$

$\alpha=0.27$

$\alpha=0.05$

Figure 3. Plots of the dimensionless average prey density for the May and RosenzweigMacArthur models at the edge and in the middle of the good patch for varying values of good patch preference. Patch sizes are varied such that: $1<l_{g}<19$ and $l_{b}=20$, and $\alpha$ was taken to be $0.05,0.25$ and 0.5 . Note that $\alpha=0.5$ corresponds to no patch preference (the standard model with continuous population density across the interface) and $\alpha=0.05$ corresponds to a strong preference for the good patch. Prey and predator had the same value of $\alpha$. We take $D_{n 1}=D_{n 2}=1.5$ and $D_{p 1}=D_{p 2}=2.1$. Other parameters are listed in Table 1.

preference leads to a drop in population density within the good patch for the Rosenzweig-MacArthur model, little difference in the middle of the good patch for the May model, and an increase in population density at the edge of the good patch for the May model.

Prey density is plotted against time in Figure 4. For the May model, we observe that changes in $\alpha$ have very little effect on the population in the middle of the good patch, and a strong effect on the population at the edge of the good patch. The edge population increases as patch preference increases. For the Rosenzweig-MacArthur model, the behaviour is quite different: The population in the middle of the patch shows a much stronger response to changes in $\alpha$ than the population at the edge of the patch. Also, in contrast to the May model both the Rosenzweig-MacArthur edge and middle populations decrease as patch preference increases. 

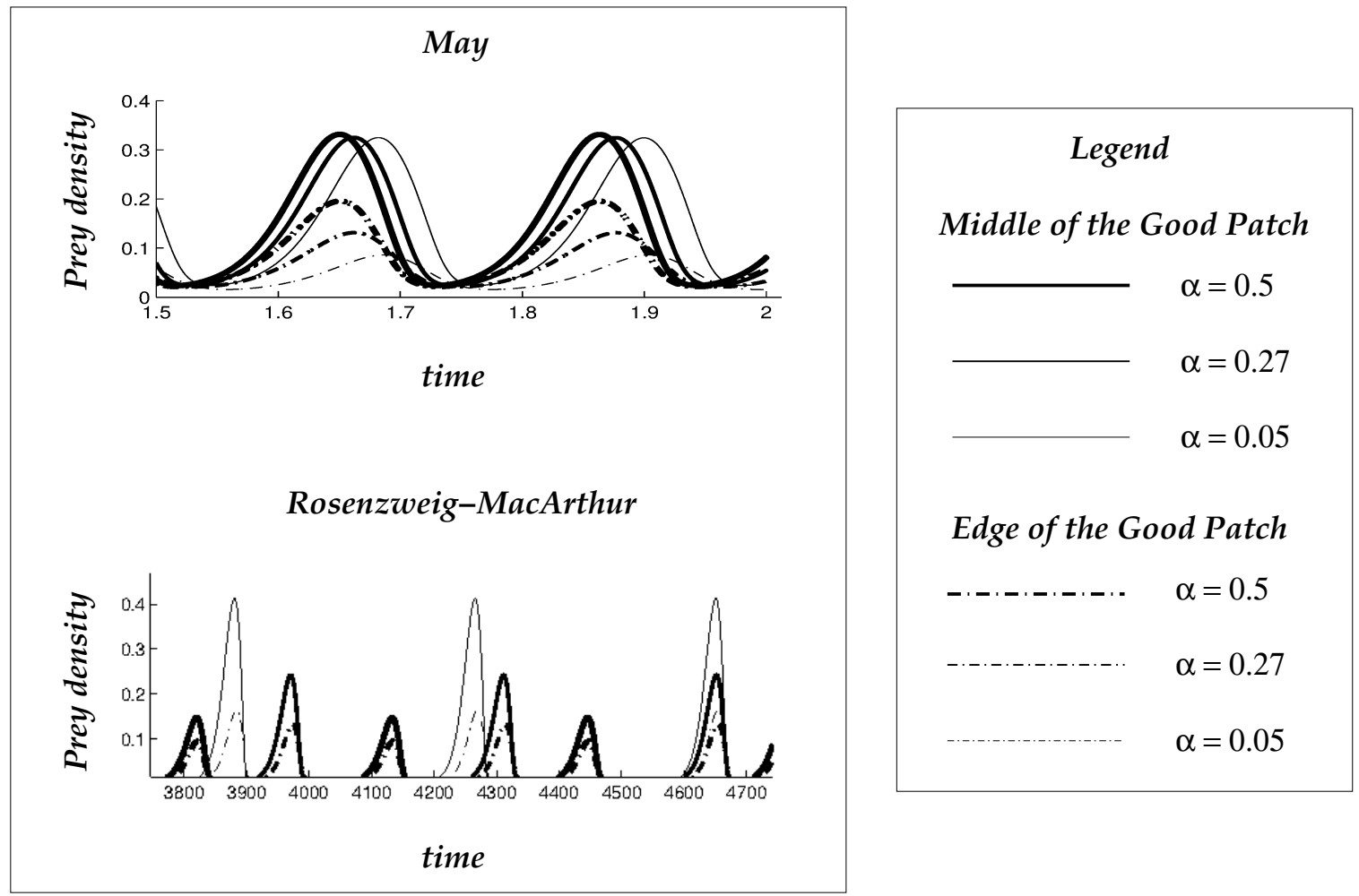

Figure 4. Plots of the dimensionless average prey density for the May and RosenzweigMacArthur models as a function of time at the edge and in the middle of the good patch for varying values of good patch preference. Good patch size $\left(l_{g}=190\right.$ for the may model and $l_{g}=70$ for the Rosenzweig-MacArthur model) was large compared to bad patch size $\left(l_{b}=20\right)$, and $\alpha$ was taken to be $0.05,0.25$ and 0.5 . Note that $\alpha=0.5$ corresponds to no patch preference (the standard model with continuous population density across the interface) and $\alpha=0.05$ corresponds to a strong preference for the good patch. We take $D_{n 1}=D_{n 2}=1.5$ and $D_{p 1}=D_{p 2}=2.1$. Other parameters are listed in Table 1.

\subsection{Habitat-dependent movement rates, $D_{1}$ and $D_{2}$}

In Section 4.2, we explored the effect of habitat loss and varying degress of habitat preference when the diffusion constant for each species was not dependent on habitat type, that is $D_{1}=D_{2}$ for both prey and predator. This allowed us to examine the effects of edge behaviours without the counfounding effects of heterogeneous responses to habitats. As mentioned in the introduction however, studies have revealed habitat-dependent movement rates for a wide variety of organisms, and so the case where $D_{1} \neq D_{2}$ is highly relevant. Here we briefly consider the effect of habitat-dependent variations in diffusion constants. Results are shown in Figure 5. Again, only the prey average density is shown, as the predator average density, prey amplitude, and predator amplitude showed similar behaviour.

We compare Figure 5 with the $\alpha=0.27$ curve in Figure 3. With increased diffusion the increase in predator density with decreasing good patch size becomes more pronounced at the edge of the patch in the Rosenzweig-MacArthur model. Aside from this difference however, the Rosenzweig-MacArthur model results are not strongly affected by the changes in movement rate. The May model, on the other hand, shows a stronger response to variations in diffusion rate. Increasing diffusion corresponds to a drop in prey density, both at the edge and in the middle of the good patch. 

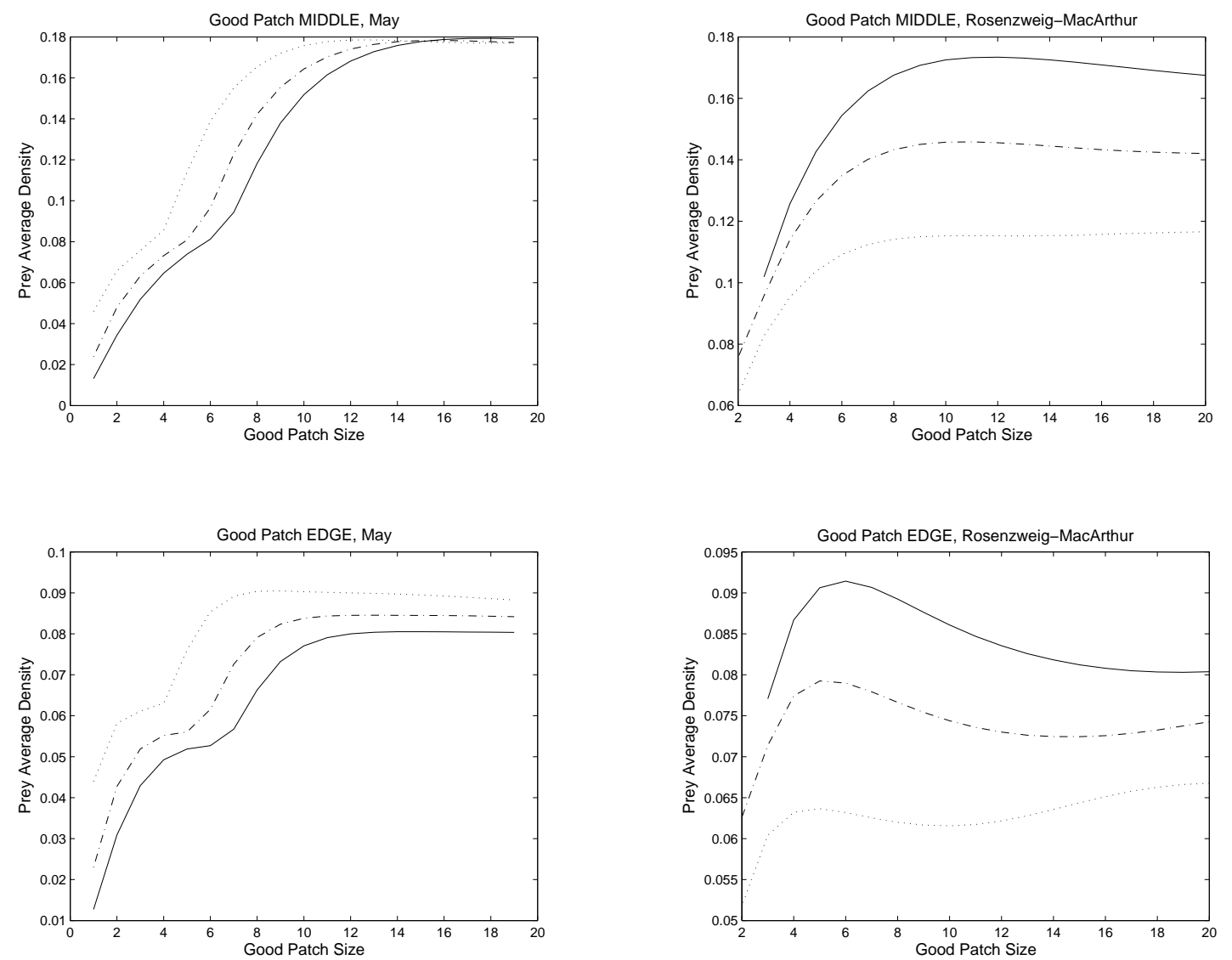

$$
D_{n 2}=3.5, D_{p 2}=4.1
$$

$$
D_{n 2}=1.5, D_{p 2}=2.1
$$

Figure 5. Plots of the dimensionless average prey density for the May and RosenzweigMacArthur models at the edge and in the middle of the good patch for varying relative movement rates in the good and bad habitat patches. Patch sizes are varied such that: $1<l_{g}<19$ and $l_{b}=20$, and the relative difference between movement rates $D$ in the good and bad patches (numbered 2 and 1 respectively) was taken to be 0,1 and 2 for both prey and predator, with movement rates being larger in the good habitat patch. Prey and predator both had the same intermediate patch preference, $\alpha=0.25$. We take $D_{n 1}=1.5$ and $D_{p 1}=2.1$ throughout. Other parameters are listed in Table 1.

\section{Results Part II: Prey and Predator Independent Movement Patterns}

\subsection{Different Patch Preference for Prey and Predator, $\alpha_{p} \neq \alpha_{n}$}

Here we relax the assumption that prey and predator have the same preference level for the good patch, and allow the two values of $\alpha$ to vary independently. The notion that preference behaviour at patch boundaries can vary between species is supported by field studies. Consider the population distribution of two sympatric Maculinea butterflies [25]: The density of Maculinea nausithous is highly positively influenced by the proportion of afforested meadow edges while Maculinea teleius shows no preference for afforested edges. In an entirely different system, recent research [13] has shown that the habitat specialist predator Canada lynx may select appropriate habitats differently than its prey, the 

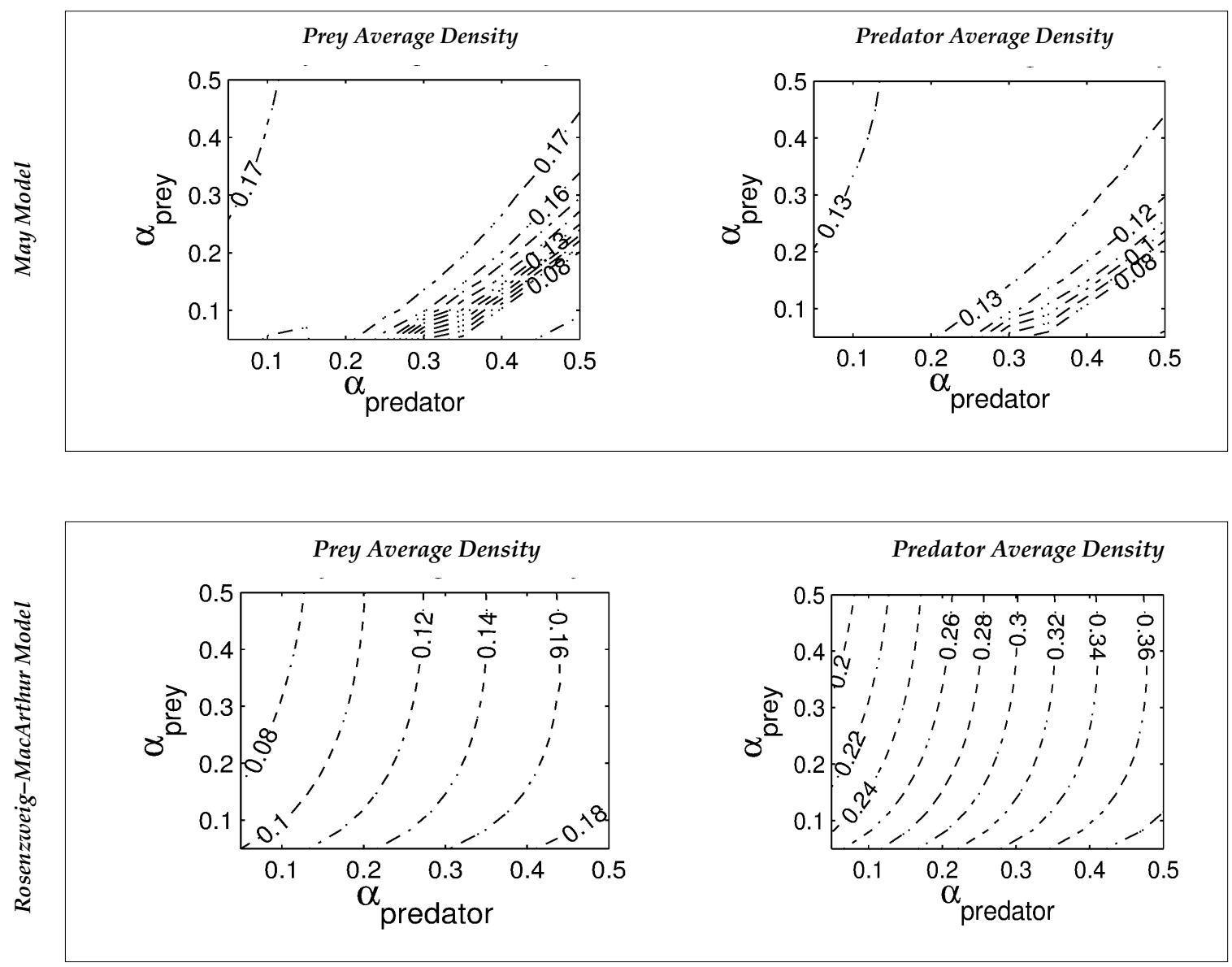

FiguRE 6. Contour plots of the dimensionless prey and predator average density, plotted in the middle of the good patch for both the May and Rosenzweig-MacArthur models. Patch sizes are: $l_{g}=l_{b}=20$. We take $D_{n 1}=D_{n 2}=1.5$ and $D_{p 1}=D_{p 2}=2.1$. Other parameters are listed in Table 1.

snowshoe hare. In this section therefore, we allow $\alpha_{\text {prey }}$ and $\alpha_{\text {predator }}$ to vary independently while patch sizes are held constant $\left(l_{g}=l_{b}=20\right)$.

The results are presented in Figures 6 and 7. For these simulations, the prey and predator showed different behaviour in response to changes in $\alpha_{\text {prey }}$ and $\alpha_{\text {predator }}$, while the prey and predator amplitudes showed the same behaviour as the prey and predator average densities, respectively. So we show the prey and predator average densities in Figures 6 and 7 .

In the middle of the good patch (Figure 6), we find the May model predator and prey average densities are relatively insensitive to changes in patch preference for the prey and the predator, except when the prey have a high preference for the good patch $\left(\alpha_{\text {prey }}\right.$ near 0$)$. In this scenario, decreases in predator preference for the good patch result in very rapid decreases in prey and predator average density in the good patch.

The Rozenzweig-MacArthur predator and prey average densities are also largely insensitive to changes in prey patch preference. In contrast to the May populations however, the prey and predator average 
Good Patch EDGE
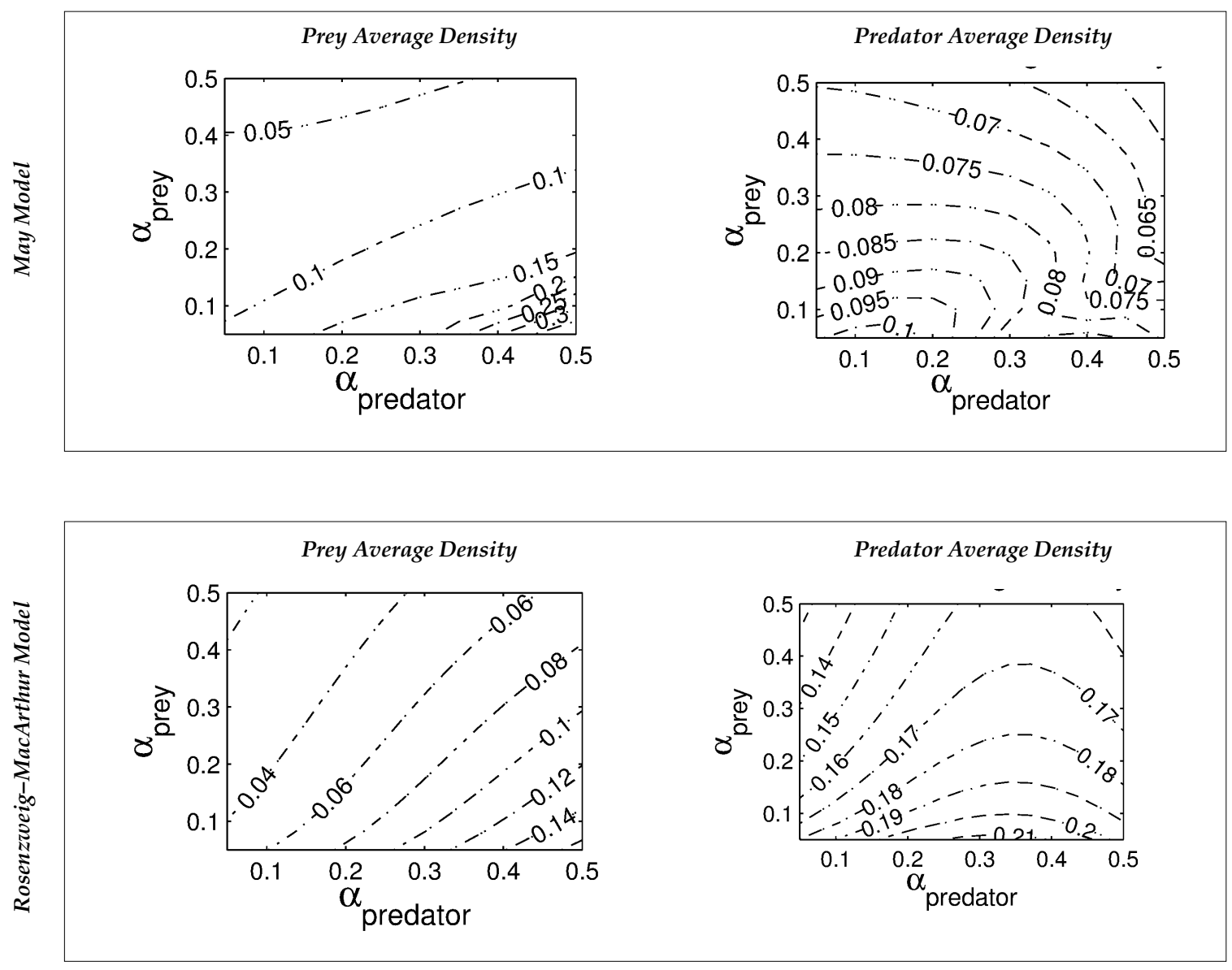

FiguRE 7. Contour plots of the dimensionless prey and predator average density, plotted at the edge of the good patch for both the May and Rosenzweig-MacArthur models. Patch sizes are: $l_{g}=l_{b}=20$. We take $D_{n 1}=D_{n 2}=1.5$ and $D_{p 1}=D_{p 2}=2.1$. Other parameters are listed in Table 1.

densities change almost linearly with changes in predator patch preference. Both densities increase as predator preference for the good patch decreases.

At the edge of the good patch (Figure 7), we find that there are more similarities in the behaviour of the two models. Prey average density increases as prey patch preference increases while predator patch preference decreases. The decrease is more abrupt for the May model than for the Rosenzweig-MacArthur model. Predator average density depends on patch preference in a more complicated way. As with the prey, for both models the predator average density is highest when prey patch preference in highest $\left(\alpha_{\text {prey }}\right.$ near 0 ). The dependence on predator patch preference is non-monotonic, with predator average density first increasing and then decreasing as predator patch preference decreases $\left(\alpha_{\text {predator }}\right.$ approaches $\left.1 / 2\right)$. The increase is more marked for the Rosenzweig-MacArthur model than for the May model. 


\subsection{Fixed Jumps in Population Density, $\kappa$}

In this section, we explore how an observed difference in population density across an interface might change in response to fragmentation under the two different scenarios explored in Parts I and II (constant movement rates between patches and constant patch preference between predator and prey).

The value of the parameter $\kappa$ is defined in (2.13). It is a result of both the patch preference behaviour at edges, and of the movement behaviour in different habitats. That is, $\kappa$ depends on both $\alpha$ and $D_{2} / D_{1}$. The population distribution that arises as a result of the edge-dependent and habitat-dependent movement behaviours has a discontinuous jump in density that is described by $\kappa$. In other words, $\kappa$ is a measure of the discontinuity in population density at the interface between habitats, resulting from the patch preference choices made at the interface between patches and the movement rates within each patch.

When the diffusion constants are habitat-independent (and therefore equal), $\kappa$ depends only on $\alpha$. This is the case we have considered thus far. When the diffusion constants are also variable across the interface, a given value of $\kappa$ can be achieved either by varying patch preference at the interface, or by varying within-patch movement rates. Two very different behaviours therefore, can result in the same jump in population density at the interface. Mathematically, we observe that a given jump in population density, as determined by $\kappa$ in (2.13), can be defined by either $\alpha$ (with the ratio $D_{2} / D_{1}$ fixed at 1 ),

$$
k=\frac{\alpha}{1-\alpha},
$$

or the ratio $D_{2} / D_{1}$ (with $\alpha$ fixed at 0.5 ),

$$
k=\frac{D_{2}}{D_{1}} .
$$

Note that a given value of $\kappa$ can be obtained for an infinite number of different combinations of the ratio $D_{2} / D_{1}$ and $\alpha$; we concentrate here on the two extremes defined in (5.1) and (5.2).

\begin{tabular}{|c||c|c|c|}
\hline $\begin{array}{c}\text { fixed population density } \\
\text { jump size }\end{array}$ & \multicolumn{2}{|c|}{$\begin{array}{c}\kappa \text { dependent on } \\
\text { movement rates, see (5.2) }\end{array}$} & $\begin{array}{c}\kappa \text { dependent on } \\
\text { patch preference, see (5.1) }\end{array}$ \\
\hline \hline$\kappa$ & $D_{n 1}$ & $D_{p 1}$ & $\alpha$ \\
\hline $0.89-0.90$ & 1.66 & 2.33 & 0.47 \\
$0.67-0.69$ & 2.18 & 3.05 & 0.4 \\
$0.47-0.48$ & 3.16 & 4.42 & 0.32 \\
0.26 & 5.7 & 8 & 0.21 \\
0.05 & 30 & 42 & 0.05 \\
\hline
\end{tabular}

TABLE 2. Parameter values used in the simulations comparing population dynamics for a fixed population density jump size, $\kappa$, when that jump occurs as a result of patch preference behaviour at habitat edges ( $\kappa$ defined by (5.1)), and when the jump occurs as a result of habitat-dependent movement rates $(\kappa$ defined by $(5.2))$. In the first case, diffusion rates in the good patch were held constant at $D_{n 2}=1.5$ for the prey and at $D_{p 2}=2.1$ for the predator. Diffusion rates in the bad patch were larger, consistent with the idea that organisms disperse more quickly in unfavourable habitats. Patch sizes were held constant at $l_{g}=l_{b}=20$.

In these simulations we fixed $\kappa$, and compared the predator-prey dynamics obtained by varying patch preference with those obtained by varying movement rates. For each predator-prey model, pairs of simulations were run for $0<k<1$. In the first set of simulations, the $\kappa$ range was obtained by varying patch preference while movement rates were held equal as in (5.1). In the second set of simulations, the same $\kappa$ range was obtained by varying movement rates in the absence of any patch preference as in (5.2). The domain size was held constant at $l_{g}=l_{b}=20$. The parameter values used are given in Table 2 . 

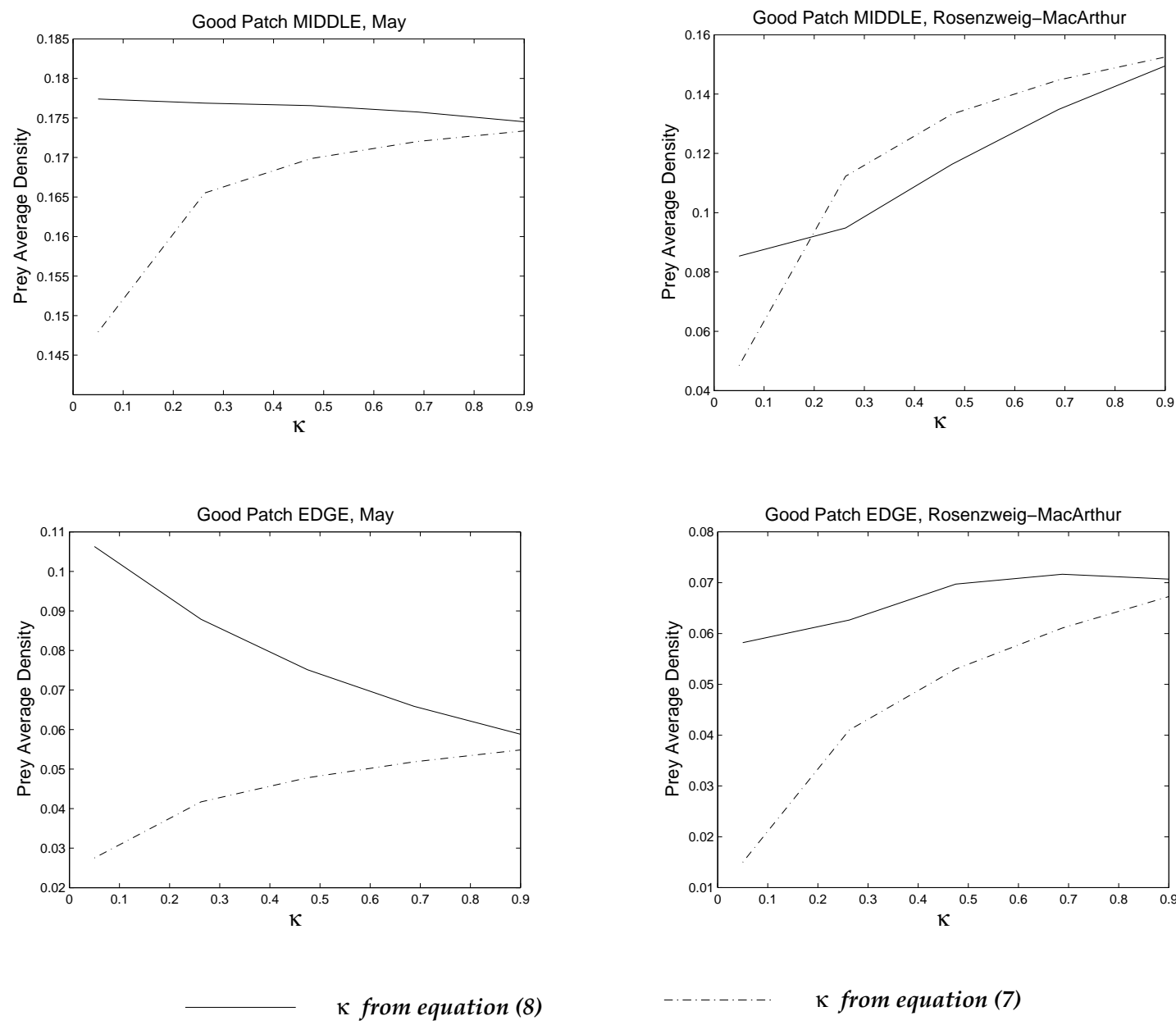

$\kappa$ from equation (7)

FiguRE 8. Plots of the dimensionless prey density against $\kappa$, the observed jump in population density across the patch interface. Density is plotted in the middle of the bad patch and at the edge of the good patch. Parameter values are listed in Table 2.

As in Sections 4.2 and 4.3, we measured prey and predator average density over a full period, and the amplitude in prey and predator density. The results are shown in Figure 8. Again, only the prey average density results are shown.

When movement rates are habitat-independent, $D_{1}=D_{2}$ for each species, and patch preference behaviour is exhibited at the interface between patches, $\kappa$ is given by equation 5.1. In this case, the higher the increase (jump) in population density from the bad patch to the good one, the higher the population density both at the edge and in the middle of the good patch. In contrast, when there is no preference behaviour exhibited at the patch edge, and movement rates are habitat-dependent, the response of the edge and middle populations is different both within and between models. In this case, $\kappa$ is given by (5.2), that is, it depends entirely on the ratio of movement rates between patches. In this case, higher jumps in population density do not necessarily correspond to higher population densities within the good patch. In particular, for the edge population in the May model, larger jumps in population density across the bad-good patch interface correspond to strong decreases in population density. The middle May population also shows a decrease, albeit a much smaller one. 


\section{Discussion}

In this paper, we examined the effect of habitat fragmentation on cyclic predator-prey populations when those populations respond either to patch edges between contrasting habitats, independently of the way they move within patches, or have movement rates that are dependent on the habitat. Our goal was to understand how variations in movement patterns affect the manner in which the cyclic dynamics change as good patch size decreases.

To keep our study general, we investigated the effect of habitat fragmentation in the context of two models: The May and Rosenzweig-MacArthur models. These two models are both simple, but plausible and well-known mathematical representations of real predator-prey interactions. While both models share the same prey equation, the differences in the predator equations result in significant differences in the underlying dynamical structures of the two models [30]. Our study therefore, explores a wide range of possible responses to habitat fragmentation due to variations in both behaviour and model structure. In particular, our study sheds light on the interplay between habitat fragmentation and habitat preference and their overall effect on the population dynamics of cyclic predator-prey systems.

To characterize changes in the cyclic dynamics, we used parameter values that gave the same cyclic dynamics from each model, in terms of five cycle probes: prey and predator maximum and minimum densities and cycle period. Our baseline for comparison then, was two models giving approximately the same cyclic dynamics in the absence of spatial effects. We then observed how the two models responded to changes in movement rates in a spatial domain with one good patch surrounded by two bad patches.

A number of interesting behaviours emerge from our study. For the Rosenzweig-MacArthur model, increasing preference for the good habitat patch results in a decrease in population density (for both prey and predator) for a given good patch size. Thus, any benefit to population growth that is gained by the prey population, is counteracted by the increased predation caused by the predator preference for the good patch. At the same time however, the prey and predator cycle amplitude decreases (since these variables follow the same pattern as prey density). So there is an increase in stability (smaller oscillations) associated with the increase in patch preference.

For the May model, the opposite behaviour is observed, with population density (and amplitude) increasing at the edge of the patch as good patch preference increases. The contrast in behaviour between the two models was unexpected, and we do not currently have a satisfactory explanation for the phenomenon. Arguing heuristically, increased patch preference should correspond to a decreased loss rate for predators especially, and therefore increased predation in the good patch, especially at the edges. Increased predation, if it is not too large, should destabilize the predator-prey system, leading to larger oscillations. This is what we see in the May populations at the edge of the patch, but we observe the opposite with the Rosenzweig-MacArthur populations. So clearly the spatial component interacts with the limit cycle predator-prey dynamics in a nontrivial way, and more analysis is required to determine the reasons for these differences in behaviour.

The May population in the middle of the patch is less sensitive to changes in patch preference than the Rosenzweig-MacArthur model, suggesting that edge effects propagate more easily in the RosenzweigMacArthur model. This may be due to differences in the structure of the limit cycle in each model. The nonspatial Rosenzweig-MacArthur limit cycle tends to have very low minima in both prey and predator, and these minimum densities persist for a significant fraction of the population cycle (Figure 4). Thus, small changes in population densities at patch edges could be relatively large in comparison to the minimum populations there. Subsequently, there is a disproportionately large effect on the populations in the middle of the good patch.

Both models, as expected, show a precipitous decrease in average density and cycle amplitude once good patch size becomes sufficiently small, consistent with earlier work [29]. Before that minimum patch size is reached however, both models show, for at least some populations, an initial increase in population density as good patch size decreases, also observed for some populations in earlier work [29]. This behaviour is diametrically opposed to the overall behaviour, and thus deserves some explanation. The strongest increases in population density with decreasing good patch size are seen in the Rosenzweig- 
MacArthur model, and a slight increase is observed in the May population in the middle of the good patch. Decreasing good patch size should correspond to increased loss of prey and predators through the interface and into the bad patch, with the loss being larger for the more rapidly-moving predator. A more rapid loss of predators should translate into decreased predation, allowing the prey to increase, with the predators eventually increasing also. While this argument is compelling, an increased predator death rate in the non-spatial models should stabilize the system, moving it into a regime of smaller oscillations and lower population averages. So again, it is unclear what exactly is driving the observed behaviour.

We note here that we obtained this behaviour, that is, increasing population density and amplitude with reduction in good patch size, for parameter values that give us cycle characteristics that are a reasonable approximation of those seen in the snowshoe hare and Canada lynx population cycles [29,31]. This observation suggests that the regime where populations can initially show, on average, an increase in density with decreasing good patch size, is potentially realistic. Critically, the population density increases that we observe in our simulations peak immediately before a rapid drop in both populations toward extinction. Caution must thus be exerted in interpreting the meaning of favourable changes in population densities as fragmentation is increased.

One possible key to the differences between the May and Rosenzweig-MacArthur model responses is the manner in which predator growth and death is handled in the two models. In the Rosenzweig-MacArthur model, there is a constant per capita death rate, irrespective of the prey population density. The prey population only affects the growth rate of the predator. In the May model, there is a constant per capita growth rate, and a density-dependent death rate that depends on both the prey and predator densities. If these differences are the main factors underlying the differences in model behaviours, caution must therefore be exercised in applying any of these models to real systems and predicting their response to environmental disturbances. Our conclusion is a warning to modelers akin to the work by Fussmann and Blasius [8].

Another possible explanation for the differences between the two model responses, is the size of the prey growth rate parameter, $r$. A larger prey growth rate should mean that the prey population is less vulnerable to decreasing good patch size. We completed some preliminary investigations of this idea (results not shown), but the results were inconclusive and for the $r$ values that we investigated the differences between the two models did not decrease.

Previous results from Maciel and Lutscher [17] stated that high patch preference (or fast movement in bad patches) in a single population corresponds to a lower minimal patch size. Our results from the May model support this conclusion, since prey and predator with high patch preference were very resistant to small good patch sizes. As the Rosenzweig-MacArthur model did not reach a critical patch size in our study we were unable to compare to the results of Maciel and Lutscher.

We also found that varying habitat-dependent movement rates (changes in the within-patch diffusion coefficients) can yield results that are very different from those obtained using changes in edge-dependent preferences (at the interface between habitats) resulting in a given population discontinuity (as defined by the parameter $\kappa$ ). This observation is in accordance with the predictions of Maciel and Lutscher [17], who state that these two behavioural patterns cannot have the same effect, as the diffusion coefficients affect the population flux at the interface, whereas $\alpha$ does not.

Our results from the Rosenzweig-MacArthur model predict a decrease in average density and amplitude of oscillations when the predator has strong preference for suitable habitat patches (which are defined as patches where prey can reproduce $[18,21]$ ). Assuming that habitat preference behaviour is more pronounced when neighbouring habitats are very different and distinguished by a hard (or narrow) edge, our result suggests that average density and amplitude may be smaller in areas where patches of suitable habitat exist in a landscape composed of a mosaic of high contrast habitat patches. This result is consistent with the observation that many boreal cyclic populations exhibit significantly damped population cycles in the southern parts of their range where forestry activities are more intense [29].

The many differences we observe in the responses of the two predator-prey systems indicate that the interplay between behaviour and habitat fragmentation is very complex in systems with cyclic dynamics. 
Given the number of organisms exhibiting cyclic dynamics, it is important that we refine our understanding of these systems. The urgency of this task is further increased when one considers that a large number of cyclic species occur in the boreal forest [16], and these species are under immediate and extensive threat from climate change and increased harvesting in the north. Future work is needed to illuminate exactly how patch preference behaviour and habitat-dependent movement rates change the dynamics of cyclic systems, and to investigate their behaviour as patch sizes become very small.

We note here that we only examined one type of habitat fragmentation in this paper, that is, decrease of the size of a good patch when the size of each bad patch is held constant. In a fragmented domain of good and bad patches, with size $l_{g}$ (Good Patch Size) and $l_{b}$ (Bad Patch Size), three types of fragmentation can be described $[7,29]$. Habitat loss is the decrease of $l_{g}$ while $l_{b}$ is held constant. Habitat separation is the increase of $l_{b}$ while $l_{g}$ is held constant. Habitat loss and separation combined is the increase of $l_{b}$ and the simultaneous decrease of $l_{g}$ while the size of the whole domain is held constant. In earlier work on cyclic populations in the presence of habitat fragmentation [29], all three types of fragmentation were investigated, and habitat loss had the strongest effect. We also briefly investigated all three types of fragmentation, and found that our results were consistent with the earlier work, and so we only presented here the results of our habitat loss simulations.

The study presented here is a preliminary investigation of the interplay between patch preference behaviour, habitat fragmentation, and cyclic population dynamics in a very simple linear domain composed of two bad patches surrounding a central good patch. Patch "goodness" is defined simply in terms of the growth rate of the prey: Prey growth is positive in good patches and zero in bad patches. More natural systems however, have a range of patch qualities and associated growth rates $[10,18]$. In our simulations we considered a "good" patch that was uniformly of high quality throughout. An important question remaining then, is how variations in quality within the good patch will modify the results obtained here.

Acknowledgements. The authors gratefully acknowledge Frithof Lutscher for helpful discussions, and the students in the Tyson lab for valuable feedback on the research project and manuscript. Two thorough reviewers also provided very constructive feedback which has improved the manuscript considerably. This research was supported by NSERC, through the Discovery Grants program, and the UBC Okanagan Institute for Species at Risk and Habitat Studies.

\section{References}

[1] H.R. Akcakaya, Population cycles of mammals: evidence for a ratio-dependent hypothesis, Ecological Monographs, 62 (1992), No. 1, 119-142.

[2] A. Blässle, Stochastic Modelling of Animal Movement using Intermittent Search Patterns, Master's thesis, University of British Columbia, Kelowna, BC, Canada, 2013.

[3] D.S. Chapman, C. Dytham, G.S. Oxford, Landscape and fine-scale movements of a leaf beetle: The importance of boundary behaviour, Oecologia, 154 (2007), No. 1, 55-64.

[4] A. Costa, A. Min, C.K. Boone, A.P. Kendrick, R.J. Murphy, W.C. Sharpee, K.F. Raffa, J.D. Reeve, Dispersal and edge behaviour of bark beetles and predators inhabiting red pine plantations, Agricultural and Forest Entomology, 15 (2013), No. 1, 1-11.

[5] J.T. Cronin, Habitat edges, within-patch dispersion of hosts, and parasitoid oviposition behavior, Ecology, 90 (2009), No. 1, 196-207.

[6] L. Edelstein-Keshet, Mathematical models in biology, Society for Industrial and Applied Mathematics, Philadelphia, 2005.

[7] L. Fahrig, Effects of habitat fragmentation on biodiversity, Annual Review of Ecology Evolution and Systematics, 34 (2003), 487-515.

[8] G.F. Fussmann, B. Blasius, Community response to enrichment is highly sensitive to model structure, Biology Letters, 1 (2005), 9-12.

[9] E. Gurarie, J. Suutarinen, I. Kojola, O. Ovaskainen, Summer movements, predation and habitat use of wolves in human modified boreal forests, Oecologia, 165 (2011), 891-903.

[10] D.L. Haughland, K.W. Larsen, Exploration correlates with settlement: Red squirrel dispersal in contrasting habitats, Journal of Animal Ecology, 73 (2004), 1024-1034.

[11] R.M. Inman, M.L. Packyla, K.H. Inman, A.J. Mccue, G.C. White, J. Persson, B.C. Aber, M.L. Orme, K.L. Alt, S.L. Kain, J.A. Fredrick, B.J. Oakleaf, S.S. Sartorius, Spacial ecology of wolverines at the southern periphery of distribution, The Journal of Wildlife Management, 76 (2012), No. 4, 778-792. 
[12] H.B. Jackson, K.A. Baum, T. Robert, J.T. Cronin, Habitat-Specific Movement and Edge-Mediated Behavior of the Saproxylic Insect Odontotaenius disjunctus (Coleoptera: Passalidae), Environmental Entomology, 38 (2009), No. 5, 1411-1422.

[13] J.L. Keim, P.D. DeWitt, S.R. Lele, Predators choose prey over prey habitats: evidence from a lynx-hare system, Ecological Applications, 21 (2011), No. 4, 1011-1016.

[14] G.M. Koehler, B.T. Maletzke, J.A. Von Kienast, K.B. Aubry, R.B. Wielgus, R.H. Naney, Habitat fragmentation and the persistence of lynx populations in Washington State, Journal of Wildlife Management, 72 (2008), No. 7, $1518-1524$.

[15] M. Kot, Elements of mathematical ecology, Cambridge University Press, Cambridge, U.K., New York, 2001.

[16] C.J. Krebs, S. Boutin, R. Boonstra (eds.), Ecosystem dynamics of the boreal forest: The kluane project, Oxford University Press, Oxford; New York, 2001.

[17] G.A. Maciel, F. Lutscher, How individual movement response to habitat edges affects population persistence and spatial spread, American Naturalist, 182 (2013), No. 1, 42-52.

[18] N.P. McCann, R.A. Moen, Mapping potential core areas for lynx (lynx canadensis) using pellet counts from snowshoe hares (lepus americanus) and satellite imagery, Canadian Journal of Zoology, 89 (2011), 509-516.

[19] H.W. McKenzie, Linear features impact predator-prey encounters: Analysis with first passage time, Master's thesis, University of Alberta, Edmonton, AB, Canada, 2006.

[20] W.W. Murdoch, B.E. Kendall, R.M. Nisbet, C.J. Briggs, E. McCauley, R. Bolser, Single-species models for manyspecies food webs, Nature, 417 (2002), 541-543.

[21] D.L. Murray, T.D. Steury, J.D. Roth, Assessment of canada lynx research and conservation needsin the southern range: another kick at the cat, Journal of Wildlife Management, 72 (2008), No. 7, 1463-1472.

[22] J.D. Murray, Mathematical biology, Springer-Verlag, Berlin, New York, 1989.

[23] O. Ovaskainen, S.J. Cornell, Biased movement at a boundary and conditional occupancy times for diffusion processes, Journal of Applied Probability, 40 (2003), 557-580.

[24] J.D. Reeve, J.T. Cronin, Edge behaviour in a minute parasitic wasp, Journal of Animal Ecology, 79 (2010), No. 2, $483-490$

[25] L. Ries, D.M. Debinski, Butterfly responses to habitat edges in the highly fragmented prairies of central Iowa, Journal of Animal Ecology, 70 (2001), 840-852.

[26] E.K. Rueness, N.C. Stenseth, M. O’Donoghue, S. Boutin, H. Ellegren, K.S. Jakobsen, Ecological and genetic spacial structuring in the Canadian lynx, Nature, 425 (2003), No. 6953, 69-72.

[27] K.L. Ryall, L. Fahrig, Response of predators to loss and fragmentation of prey habitat: A review of theory, Ecology, 87 (2006), No. 5, 1086-1093.

[28] S. Senger, R. Tyson, B.D. Roitberg, H.M.A. Thistlewood, A.S. Harestasd, M.T. Chandler, Influence of habitat structure and resource availability on the movements of rhagoletis indifferens (Diptera: Tephritidae, Environmental Entomology, 38 (2009), 823-835.

[29] S. Strohm, R. Tyson, The effect of habitat fragmentation on cyclic population dynamics: A numerical study, Bulletin of Mathematical Biology, 71 (2009), 1323-1348.

[30] S. Strohm, R.C. Tyson, The effect of habitat fragmentation on cyclic population dynamics: A reduction to ordinary differential equations, Theoretical Ecology, 5 (2011), No. 4, 495-516.

[31] R. Tyson, S. Haines, K.E. Hodges, Modelling the canada lynx and snowshoe hare lpopulation cycle: The role of specialist predators, Theoretical Ecology, 3 (2010), 97-111.

[32] R. Tyson, H. Thistlewood, G.J.R. Judd, Modelling dispersal of sterile male codling moths, cydia pomonella, across orchard boundaries, Ecological Modelling, 205 (2007), 1-12.

[33] N.O. Villis, Shape of patch edges affects edge permeability for meadow voles, Ecological Applications, 22 (2012), No. 6, $1827-1837$.

[34] H. Ylonen, R. Pech, S. Davis, Heterogeneous landscapes and the role of refuge on the population dynamics of a specialist predator and its prey, Bulletin of Mathematical Biology, 59 (2003), 107-137. 